\section{Immunohistochemical LRIG3 expression in cervical intraepithelial neoplasia and invasive squamous cell cervical cancer: association with expression of tumor markers, hormones, high-risk HPV-infection, smoking and patient outcome}

\author{
Annika K. Lindström, ${ }^{1,2}$ Dan Hellberg ${ }^{1,2}$ \\ ${ }^{1}$ Center for Clinical Research, Falun \\ 'Department of Women's and Children's \\ Health, Uppsala University, Sweden
}

\section{Abstract}

The novel biomarker LRIG3 is a member of the LRIG family (LRIG1-3). While LRIG1 has been associated with favorable prognosis and LRIG2 with poor prognosis in invasive cervical cancer, little is known about the role of LRIG3. The aim of this study was to investigate the expression of LRIG3 in invasive cancer and cervical intraepithelial neoplasia (CIN) for possible correlation with other tumor markers, to hormones and smoking, as a diagnostic adjunct in CIN, and prognostic value in invasive cancer. Cervical biopsies from 129 patients with invasive squamous cell carcinoma and 170 biopsies showing low grade and high grade CIN, or normal epithelium were stained for LRIG3 and 17 additional tumor markers. Among other variables the following were included: smoking habits, hormonal contraceptive use, serum progesterone, serum estradiol, high-risk HPV-infection, menopausal status and ten-year survival. In CIN, high expression of the tumor suppressors retinoblastoma protein, p53, and p 16 , and Ecadherin (cell-cell interaction), or low expression of CK10, correlated to LRIG3 expression. In addition, progestogenic contraceptive use correlated to high expression of LRIG3. In invasive cancer there was a correlation between expression of the major tumor promoter c-myc and high LRIG3 expression. High LRIG3 expression correlated significantly to presence of high-risk HPV infection in patients with normal epithelium and CIN. There was no correlation between LRIG3 expression and 10year survival in patients with invasive cell cervical cancer. LRIG3 expression is associated with a number of molecular events in CIN. Expression also correlates to hormonal contraceptive use. The results on expression of other tumor markers suggest that LRIG3 is influenced by or influences a pattern of tumor markers in cancer and precancerous cells. Further studies are needed to elucidate if LRIG3 expression might be clinically useful.

\section{Introduction}

The human leucine-rich repeats and immunoglobulin-like domains (LRIG) protein family comprises integral plasma membrane proteins LRIG1, 2, and $3 .{ }^{1}$ LRIG1 negatively regulates growth factor signalling and is a proposed tumor suppressor. ${ }^{2}$ In early stage uterine cervical carcinoma, expression of LRIG1 has been associated with good survival ${ }^{3}$ and LRIG2 expression appeared to be a significant predictor of poor prognosis in one study. ${ }^{4}$ LRIG1 and LRIG2 expression was observed in normal epithelium and in precancerous cervical epithelium [cervical intraepithelial neoplasia (CIN)] and found to increase with increasing grade. ${ }^{5}$ Less is known about the function and expression of LRIG3 and it has not been studied in squamous cell cervical carcinoma (SCC) or CIN previously. The protein encoded by the LRIG3 gene is a single chain transmembrane glycol-protein, composed by an extracellular part with a leucine-rich repeats domain, three immunoglobulin-like domains, and an intracellular domain. ${ }^{1}$ LRIG3 is located at chromosome12q13.2, which is a site susceptible to gene deletions that are related to the development of a variety of tumors. ${ }^{6-8}$ All previously investigated human tissues express LRIG3 mRNA to varying degrees. The highest expression has been seen in stomach, skin and thyroid, and low expression in heart and blood. ${ }^{1}$ The expression pattern and function of the LRIG3 gene in human tumors is not fully clarified. Data on LRIG3 expression and the association with clinic-pathological features of human cancers is still rare.

The purpose of the present study was to evaluate the expression of the LRIG3 protein in normal cervical tissue, CIN, and invasive squamous cell carcinoma, and to investigate the expression of LRIG3 and possible interactions with other tumor markers, high-risk HPV (HRHPV) infection, correlation to hormones and smoking, and clinical outcome.

\section{Materials and Methods}

The entire study population comprised 299 prospectively recruited women; the first study group included 129 women with invasive squamous cell carcinoma who had been admitted to the Department of Gynecologic Oncology, Norrlands University Hospital, Umeå, during 1984-1990 and followed up until the mid-2000s.
Correspondence: Dr. Annika K. Lindström, Center for Clinical Research, Nissers väg 3, SE-791 82 Falun, Sweden. Tel. + 46.70.5350107.

E-mail address: annika.lindstrom@ltdalarna.se

Key words: LRIG3, cervical cancer, cervical intraepithelial neoplasia, biological markers, human papillomavirus, hormonal contraceptives, smoking.

Contributions: each author has participated equally in preparing the manuscript

Conflict of interests: the authors declare no conflict of interests.

Funding: Lions Norrland Cancer Fund, Umeå and Centre for Clinical Research, Falun, Sweden.

Acknowledgments: Associate professor Håkan Hedman, Dept. of Oncology, Umeå University for providing LRIG3 antibodies and organizing staining. Associate professor Tibor Tot, Dept. of Pathology and Clinical Cytology, Falun, for microscopically evaluation of sections on glass before tissue micro array. Associate professor Anders Lindgren, Dept. of Genetics and Pathology, Uppsala University, for expertise in evaluation of antibody-staining. Dr. Anna Asplund, Dept. of Genetics and Pathology, Uppsala University, for organizing the staining of tumor markers. Dr. Raghad Samir for involvement in the CIN study.

Received for publication: 2 July 2013.

Accepted for publication: 5 February 2014.

This work is licensed under a Creative Commons Attribution NonCommercial 3.0 License (CC BYNC 3.0).

CCCopyright A.K. Lindström and D. Hellberg, 2014 Licensee PAGEPress, Italy

European Journal of Histochemistry 2014; 58:2227 doi:10.4081/ejh.2014.2227

Clinical staging of cancer patients was made according to the International Federation of Obstetricians and Gynecologists (FIG0). ${ }^{9}$ The second study group included 142 women admitted to the Department of Obstetrics and Gynecology, Falun Hospital ,between 2005 and 2007 because of an abnormal pap smear at a routine investigation or repeated cytological findings of atypical squamous cells of undetermined significance (ASCUS). They were investigated with a diagnostic cone biopsy or by colposcopically directed punch biopsies. In addition, 28 healthy volunteers in fertile ages with normal pap smear were included. All cases were categorized as normal, CIN 1, CIN 2 , CIN 3 or as borderline (if showing some but not all of the following features: mitotic figures, vertical nuclear growth pattern, perinuclear halo, indistinct cytoplasmic border and no primitive cells in upper $1 / 3$ of squamous layer), according to published criteria. ${ }^{10} \mathrm{CIN}$ 
grade was dichotomized into high-grade lesions (HCIN) - moderate dysplasia and cancer in situ (CIN 2 and CIN 3) - and low-grade lesions (LCIN) - mild dysplasia and histologically atypical cells (CIN 1 and borderline). In $36(25.4 \%)$ of the 142 women with abnormal smear the biopsy showed normal epithelium, while 59 (41.5\%) and 47 (33.1\%) biopsies, respectively, showed low-grade CIN (LCIN), high-grade CIN (HCIN). The clinical history included menopausal status, reproductive events, smoking habits, and hormonal contraceptive use. Ten-year survival was recorded for cancer patients. Seventeen tumor markers were included in the study in addition to LRIG3. In patients with normal epithelium or CIN expression of $\mathrm{CD}^{+}$, CK10, cox-2, e-cadherin, EGFR, FHIT, IL-10, Ki-67, LRIG 1-3, p16, $\mathrm{p} 53$, and $\mathrm{Rb}$ were studied. In patients with invasive cancer, expression of CD4+, CD44, cmyc, cox-2, e-cadherin, EGFR, Ki-67, LRIG1-3, p27, p53, and VEGF were diagnosed.

The tissue micro array (TMA) used in this study and details have been reported previously. ${ }^{3,4}$ Briefly, three-micrometer sections of the original paraffin blocks were reviewed by a senior pathologist and the most representative area(s) were marked for TMA. Two-millimeter punch biopsies were taken from the original blocks corresponding to the marked area and joined into TMA paraffin blocks, containing 2430 punch biopsies. Immunohistochemical staining of LRIG3 was carried out with polyclonal rabbit antibodies against the cytoplasmic tail of the protein. ${ }^{11}$ LRIG3 antibodies were provided by the Department of Oncology, Norrlands University Hospital, Umeå. For immunohistochemistry glass slides were deparaffinized in xylene $(2 \times 15 \mathrm{~min})$, dehydrated through graded alcohols and endogenous peroxidase was blocked (using $\mathrm{H}_{2} \mathrm{O}_{2}$ in $70 \%$ ethanol). Antigen retrieval was performed using Target Retrieval Solution (TRS pH 6.0 or
pH9.0, Labvision, Fremont, CA, USA) in a decloaking chamber (Biocare Medical, Walnut Creek, CA, USA) for 4 min at $125^{\circ} \mathrm{C}$. Thereafter the slides were immunostained in the automated staining instrument, where primary antibodies and secondary reagent were each incubated for $30 \mathrm{~min}$ at room temperature (RT). Finally, the slides were incubated with diaminobenzidine (DAB) as chromogen for 10 min and counterstained with Mayers hematoxylin (Sigma-Aldrich, St Louis, M0, USA) for $15 \mathrm{~min}$. Slides were washed in distilled water for 10 min, dehydrated through graded alcohols to xylene, and mounted in Pertex organic mounting medium (Histolab, Gothenburg, Sweden). Details about LRIG and the antibodies that showed some correlation to LRIG3 are given in Table 1. The remaining 11 tumor markers did not show any correlations to LRIG3 expression and details and major functions are described elsewhere. ${ }^{4,5,12}$ The neoplastic cells showed cytoplasmic staining of LRIG3 with higher perinuclear density (Figure 1). LRIG3 antibodies were provided by the Department of Oncology, Norrlands University Hospital, Umeå. Details about the antibodies that showed some correlation to LRIG3 are given in Table 1 . The remaining 11

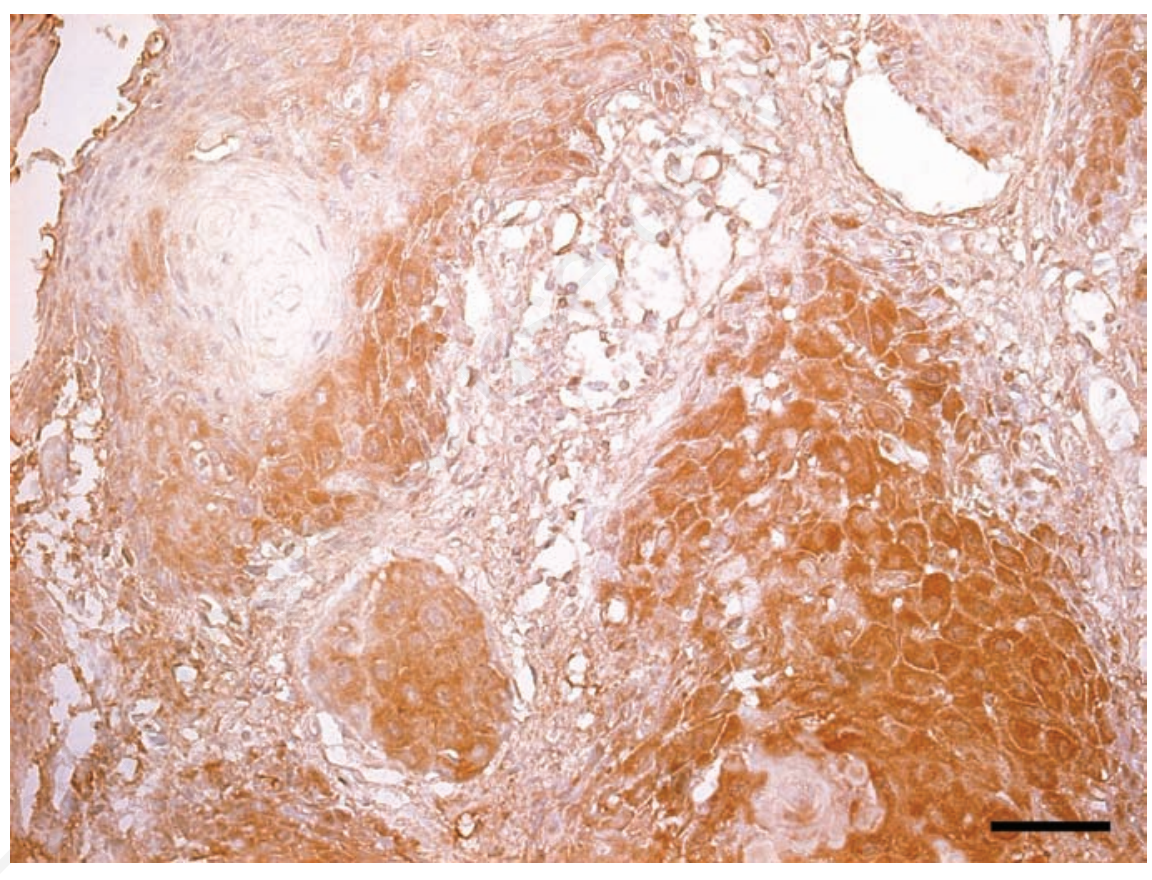

Figure 1. Immunohistochemical staining of LRIG3 in invasive squamoepithelial cervical cancer. LRIG3 staining is cytoplasmic with higher perinuclear density. Scale bar: $25 \mu \mathrm{m}$.

Table 1. Tumor markers included in the study and their major functions.

\begin{tabular}{|c|c|c|c|c|c|c|}
\hline Biological marker & Functions & Clone & Species & Dilution & $\begin{array}{l}\text { Antigen } \\
\text { retrieval }\end{array}$ & Source \\
\hline LRIG3 & Unclear (receptor regulation?) & LRIG3-207 & Rabbit & 1.50 & $\mathrm{CC} 2 \mathrm{pH} 6$ & $\begin{array}{l}\text { H Hedman Umeå } \\
\text { University, Sweden }\end{array}$ \\
\hline p53 & $\begin{array}{l}\text { Tumor suppression, } \\
\text { apoptosis }\end{array}$ & M7001 & Mouse & $1: 1000$ & HIER pH6 & DakoCytomation \\
\hline p16 & Tumor suppression & NCL-p16-432 & Mouse & 1:100 & HIER & Novocastra \\
\hline Retinoblastoma protein (Rb) & Tumor suppression & 554136 & Mouse & $1: 300$ & HIER pH9 & Pharmingen \\
\hline Cytokeratin 10 (CK 10) & Cytoskeleton & MS-611 & Mouse & 1:600 & HIER pH9 & $\begin{array}{l}\text { Lab Vision/Neo } \\
\text { Markers }\end{array}$ \\
\hline E-cadherin & Cell-cell adhesion & $13-1700$ & Mouse & $1: 2500$ & HIER pH9 & Zymed \\
\hline C-myc & $\begin{array}{l}\text { Cell cycle progression, } \\
\text { malignant transformation }\end{array}$ & 9E11 & Mouse & 1:100 & TED pH 9 DAKO & Novocastra \\
\hline
\end{tabular}


tumor markers did not show any correlations to LRIG3 expression. Expression of the 18 biomarkers included was evaluated by one external senior pathologist, who was blinded for clinical details. Frequency of stained cells and intensity of staining was diagnosed. Expression was scored as high when $20 \%$ or more of the cancer cells were immunopositive and as low when less than $20 \%$ of the cancer cells were immunopositive. This cut-off level was chosen because it showed the best explanatory power when different cut-off levels were tested $(0 \%, 1-19 \%, 20-49 \% \%$ and 50 $100 \%)$. Occasionally, it was not possible to evaluate expression of a specific tumor marker in a specific tissue. As it was not systematic in any study subject, the whole study population was included and the number of evaluations of specific tumor markers thus differed with a few observations. All women had serum progesterone and serum estradiol analyzed, as well as current hormonal contraceptive use and smoking habits. The presence of high risk HPV infection (HR-HPV) (HPV 16, 18, 31, 33, $35,39,45,51,52,56,58$, and 59) was checked with Hybrid Capture $2^{13}$ in women with normal epithelium or CIN, but not in women with SCC. ${ }^{13,14}$ For statistical analyses the JMP statis- tical package, SAS institute was used. $\chi^{2}$ test (likelihood ratio) was used in univariate analyses and logistic regression in multivariate analyses to adjust for confounding. When counts in one or more cells were less than five the P-values were checked by Fisher's exact test. Ten year survival was recorded for patients with SCC.

The study was approved by the research ethical committees in Uppsala and Umeå. Written informed consent was obtained.

\section{Results}

Mean age of women with normal histology or CIN was 36.4 years (range 18-65) and 59.7 years (range 24-89 years) in women with SCC. In the former group $15 \%$ were postmenopausal, while the corresponding figure in women with SCC was 69\%. Expression of LRIG3 could be evaluated in 119 (92.2\%) of cancer patients and in all 170 women with normal histology or CIN. In patients with SCC the clinical stage and age correlated with 10-year survival, but age was non-significant after adjustment for stage. Ten-year mortality rate was $41 \%$ and expression of LRIG 3 had no prognostic significance. In patients with normal epithelium or CIN 35.7\% were current smokers and in SCC $48 \%$ were current smokers. LRIG3 expression showed no association with smoking. There was a trend for higher LRIG3 expression in HCIN compared to LCIN and normal epithelium. High expression of LRIG3 ( $\geq 20 \%$ stained cells) was observed in $15-19 \%$ in normal epithelium and low-grade CIN compared to $29-42 \%$ in high-grade CIN (CIN 2 and CIN 3 and in invasive cancer (Table 2). High LRIG3 expression correlated significantly to the presence of HR-HPV infection compared to no HR-HPV infection $(28.3 \%$ vs $11.9 \%$, $\mathrm{P}=0.046$, not shown in Table). When LRIG3 expression was adjusted for histopathological diagnosis, i.e. normal, LCIN and HCIN, the correlation to presence of HR-HPV infection vanished $(\mathrm{P}=0.18)$. Correlation to HPV infection was not studied in patients with invasive cancer where HPV is expected to be found in close to all tumors. In CIN, Rb, p53, p16 and ECadherin expression was associated with high LRIG3 expression, while CK10 expression correlated to low LRIG3 expression. In invasive cancer there was a positive correlation between expression of major tumor promoter c-myc, and high LRIG3 expression (Table 3). In

Table 2. Expression of LRIG3 in normal cervical epithelium and CIN and invasive cervical cancer.

\begin{tabular}{|c|c|c|c|c|}
\hline \multirow[t]{2}{*}{ Total } & & \multicolumn{2}{|c|}{ LRIG 3 expression staining intensity* } & \multirow[t]{2}{*}{ P-value $^{\circ}$} \\
\hline & & High expression n (\%) & xpression n (\%) & \\
\hline \multicolumn{5}{|l|}{ CIN and normal } \\
\hline Normal & 64 & $12(18.8)$ & $52(81.3)$ & 0.06 (trend) \\
\hline Low-grade CIN" & 59 & $9(15.3)$ & $50(84.8)$ & \\
\hline High-grade $\mathrm{CIN}^{\S}$ & 47 & $16(34.0)$ & $31(66.0)$ & \\
\hline \multicolumn{5}{|c|}{ Invasive cancer $(\text { stage })^{\wedge}$} \\
\hline IB/IIA & 62 & $26(41.9)$ & $36(58.1)$ & 0.57 (trend) \\
\hline IIB & 17 & $5(29.4)$ & $12(70.6)$ & \\
\hline III-IV & 40 & $14(35.0)$ & $26(65.0)$ & \\
\hline
\end{tabular}

*Absence of or weak staining: low expression; moderate or strong staining: high expression; ${ }^{\circ}$ trend; ${ }^{\sharp}$ ASCUS or LSIL (low grade squamous epithelial lesion - corresponding to CIN 1); $\$$ high grade squamous epithelial lesion - corresponding to CIN2 or CIN3; ^^staging according to International Federation of Obstetricians and Gynecologists (FIGO).

Table 3. Correlation between LRIG3 expression and tumor marker expression in normal epithelium and CIN ( $n=170)$, and invasive cancer ( $n=119)$.

\begin{tabular}{|c|c|c|c|c|c|c|c|c|}
\hline \multirow[b]{2}{*}{$\begin{array}{l}\text { Frequency of stained } \\
\text { cells } \\
(\% \text { of cells)* }\end{array}$} & \multicolumn{5}{|c|}{$\begin{array}{l}\text { CIN and normal epithelium } \\
\text { LRIG3 expression (staining frequency) }\end{array}$} & \multicolumn{3}{|c|}{$\begin{array}{c}\text { Invasive cervical cancer } \\
\text { LRIG3 expression (staining frequency) }\end{array}$} \\
\hline & $\begin{array}{c}\text { High expression }{ }^{\circ} \\
(\mathrm{n}=37)\end{array}$ & Low & $\begin{array}{l}\text { expression }{ }^{\#} \\
(n=133)\end{array}$ & P-value & P-value ${ }^{\S}$ & $\begin{array}{c}\text { High expression }^{\circ} \\
(\mathrm{n}=45)\end{array}$ & $\begin{array}{l}\text { Low expression } \\
(n=74)\end{array}$ & P-value \\
\hline $\mathrm{Rb}(\geq 20 \%)$ & $18(51.4)$ & & $28(22.1)$ & 0.001 & 0.0009 & $\mathrm{NA}$ & NA & $\mathrm{NA}$ \\
\hline p53 ( $\geq 20 \%)$ & $13(37.1)$ & & $24(18.2)$ & 0.02 & 0.05 & $27(63.8)$ & $43(58.9)$ & 0.68 \\
\hline $\mathrm{pl6}(\geq 50 \%)$ & $18(48.7)$ & & $31(23.3)$ & 0.004 & 0.06 & $\mathrm{NA}$ & NA & $\mathrm{NA}$ \\
\hline CK10 (0\%) & $6(17.1)$ & & $49(38.6)$ & 0.01 & 0.02 & $\mathrm{NA}$ & NA & $\mathrm{NA}$ \\
\hline E-cadherin $(\geq 50 \%)$ & 32 (94.1) & & $96(74.4)$ & 0.006 & 0.02 & $37(82.2)$ & $56(80.0)$ & 0.77 \\
\hline c-myc $(>0 \%)$ & $23(22.6)$ & & $7(41.2)$ & 0.12 & 0.48 & $23(51.1)$ & $21(29.6)$ & 0.02 \\
\hline
\end{tabular}

CIN, cervical intraepithelial neoplasia; ${ }^{*} 0 \%, 1-19 \%, 20-49 \%$ and $50-100 \% ; ~{ }^{\circ}$ high expression: $\geq 20 \%$ of cells were stained by LRIG3 antibodies; ${ }^{\sharp}$ low expression: <20\% of cells were stained by LRIG3 antibodies; ${ }^{\circledR}$ adjusted for normal epithelium and CIN grade; NA, not available. 
CIN there was also a significant correlation between progestogenic hormonal contraceptives use and a high expression of LRIG3 (Table 4).

\section{Discussion}

All three LRIG proteins are expressed in many cancer types. ${ }^{2}$ In the LRIG family the function of LRIG3 is little known. Studies presented indicate LRIG1 function as a tumor suppressor. LRIG2 expression correlates to poor prognosis in early stages of cervical cancer and in some other human cancers types. ${ }^{2,4,15,16}$ Data on LRIG3 expression and the association with clinico-pathological features of human cancers are still rare. To our knowledge no data has yet been presented for the role of LRIG3 in CIN or invasive cervical cancer. In contrast to LRIG1 and LRIG2 no association between LRIG3 expression and survival was found in this study. The study only included cervical squamous cell carcinoma which has not been previously studied. In a recent study of cervical adenocarcinoma, however, both LRIG1 and LRIG3 expression was reported to be associated with favorable outcome. Only LRIG3 appeared to with favorable prognosis in multivariate analyses. ${ }^{17}$ This might seem contradictory to our results but we have previously shown that squamous cell and adenocarcinoma does not entirely show a similar tumor marker status. Thus expression of 11 tumor markers were studied and expression of six of these differed significantly between squamous cell and adenocarcinoma. None of these correlated to prognosis in adenocarcinoma, while p53, c-myc and cycloxygenase 2 (Cox-2) did; ${ }^{18}$ there was no evidence that LRIG3 expression could be used as an adjunct for diagnostic purposes. In highgrade CIN and invasive cancer expression was slightly higher $(29-42 \%)$ than in normal epithelium (19\%) and low-grade CIN (15\%) but these differences are insufficient to be used in diagnostics. In astrocytic tumors perinuclear staining of LRIG3 inversely correlated with WHO grade and significantly correlated with better survival, but is not yet confirmed. ${ }^{19}$
In vitro studies of glioblastoma cells demonstrated that RNA against LRIG3 could effectively down regulate LRIG3 gene expression, suggesting that LRIG3 might serve as a tumor suppressor gene in the pathogenesis of glioma..$^{20-22}$ On the other hand, in vitro studies in bladder cancer by Yuan et al. suggested that overexpression of the LRIG3 gene might be of procancer nature. By employing RNA interference technique they found that lowered expression of LRIG3 gene in bladder cancer cells resulted in decreased growth of bladder cells, changes in cell cycle and increased apoptosis. ${ }^{23}$ In the present study a trend was showed that expression of LRIG3 in HCIN was lower than in LCIN and normal epithelium. Expression of LRIG3 did not correlate with survival or stage in squamous cell cervical cancer which indicates that it is of less clinical importance in this cancer type. This study showed that LRIG3 expression correlated to high-risk HPV infection in normal epithelium and CIN. But when LRIG3 expression was adjusted for histopathological grade, i.e. normal, LCIN and HCIN, the correlation vanished. LRIG3 expression was high both in HPV infections and HCIN and the correlation between LRIG3 and HPV infection might merely be a confounder for CIN grade. In patients with CIN, high expression of LRIG3 correlated to progestogenic contraceptive use but there was no correlation to use of combined oral contraceptives (OC). There are some studies supporting long-term oral contraceptive use (more than 4-5 years) as risk factor for cervical cancer. $^{24,25}$ Few studies have looked into gestagenonly contraceptives and CIN, but Samir et al. found a high expression of p53 in progestogenonly users compared to non-users, and also differences in Cox-2 and $\mathrm{Rb}$ expression between progestogen-only users and OC. ${ }^{26}$ This is consistent with our results on different LRIG3 expression in these two types of hormonal contraceptives. In a phase II trial treatment with vaginal progesterone in women with LCIN a lower rate of disease regression and a higher rate of surgical interventions was observed. ${ }^{27}$ On the other hand, in a study by Darwish et al. prolonged use of progestogen-only contraceptives (more than 3 years) was not associated with increased risk of abnormal cytological findings ${ }^{28}$ in general, however, at least 5 years exposure is often defined as long-term use. The molecular role of progestogenic contraceptives is not thoroughly studied in CIN, but unfavorable prognosis and patterns of tumor markers has been reported with high serum progesterone levels. ${ }^{12,29}$ An interesting finding in CIN was that three tumor suppressors, Rb, p53 and p16 correlated to expression of LRIG3. In addition, E-Cadherin expression was associated with high, while CK10 expression correlated with low LRIG3 expression. p16 is a biomarker for transforming HPV infections that could act as an adjunct to current cytological and histological assessment of cervical smears and biopsies. Immunohistochemical analysis of p16 expression is used as a diagnostic tool. Expression is related to the degree of histological dysplasia, suggesting that it may have prognostic and predictive value in the management of cervical neoplasia. ${ }^{30}$ Although there is good evidence that p16 immunostaining correlates with the severity of cytological/histological abnormalities, the reproducibility is limited, due to insufficiently standardized interpretation of the immunostaining. ${ }^{31}$ In a recent study, p16 expression did not independently correlate to HPV positivity but correlated independently with increasing CIN grade. ${ }^{14}$ These results and our findings that high LRIG3 expression was associated with p16 expression as well as increasing CIN grade may indicate that LRIG3 could have a clinical usefulness in CIN. LRIG3 expression is probably of less use in HPV detection as both increase by increasing CIN grade. p53 is a key cell-cycle regulator and induce cell-cycle arrest at the G1 and G2 checkpoints prior to DNA replication and allowing repair of damaged DNA; ${ }^{32}$ p53 expression has been associated with good prognosis in cervical cancer. ${ }^{33}$ The positive correlation between high $\mathrm{p} 53$, as well as high $\mathrm{Rb}$ expression, and LRIG3 expression has not been previously studied. Besides $\mathrm{p} 53$, Rb is one of the classic tumor suppressors; both proteins are inactivated by HPV oncogenes. In CIN, little is known about mechanisms in tumor suppression and the correlations found in the present study warrants further research. ${ }^{34}$ In addition, there was a negative and positive cor-

Table 4. LRIG3 expression and hormonal contraceptives, sex steroid hormones, and smoking in patients with cervical intraepithelial neoplasia and normal epithelium.

\begin{tabular}{lcccc} 
& High LRIG 3 expression n (\%) & Low LRIG 3 expression n (\%) & P-value \\
Oral contraceptive use & $5(16.1)$ & $35(32.1)$ & 0.07 & 0.03 \\
Gestagens & $8(33.3)$ & $11(12.8)$ & 0.88 \\
Serum estradiol (pmol/L) & 322 & 311 & 0.9 & 0.73 \\
Serum progesterone (nmol/L) & 10.1 & $46(34.9)$ & 0.66 \\
\hline Smoking & $14(38.9)$ & & \\
\hline
\end{tabular}

Postmenopausal women were excluded in hormonal analyses. 
relation, respectively between CK10 and E-cadherin expression, and LRIG3 expression. Both proteins are rarely studied in CIN, ${ }^{14,35}$ the former involved in the cytoskeleton, the latter in cell-cell interactions. Finally, the only tumor marker that correlated to LRIG3 expression in invasive cancer was c-myc. Paradoxically, LRIG3 correlated to tumor suppressor expression in CIN, but not to c-myc, a potent oncoprotein in cervical cancer. However, LRIG3 was not a prognostic predictor, while c-myc has been incriminated in tumor progression. ${ }^{33}$ In one study a combination of c-myc and bcl expression was used to discriminate between invasive cervical cancer and earlier lesions. Co-expression was only found in invasive cancer, but the sensitivity was very low. ${ }^{36}$

In conclusion, LRIG3 did not have prognostic value as a single marker or in combination with other tumor markers studied in invasive squamous cell cervical cancer. In CIN a positive correlation with LRIG3 expression and HRHPV, use of progestogenic contraception and tumor suppressors Rb, p53 and p16 gives support that LRIG3 is involved in the development of neoplasia. The results on expression of other tumor markers suggest that LRIG3 is influenced by or influences a pattern of tumor markers in the precancerous cells. Whether LRIG3 is activated in defense of CIN development or is a promoter has not yet been clarified. Future studies will show if LRIG3 can be a candidate to be part in a panel of tumor markers in diagnosis and prognosis of CIN.

\section{References}

1. Guo D, Holmlund C, Henriksson R, Hedman $H$. The LRIG gene family has three vertebrate paralogs widely expressed in human and mouse tissues and a homolog in Ascidiacea. Genomics 2004;84: 157-65.

2. Hedman H, Henriksson R. LRIG inhibitors of growth factor signalling - double-edged swords in human cancer? Eur J Cancer 2007;43:676-82.

3. Lindstrom AK, Ekman K, Stendahl U, Tot T, Henriksson R, Hedman H, et al. LRIG1 and squamous epithelial uterine cervical cancer: correlation to prognosis, other tumor markers, sex steroid hormones, and smoking. Int J Gynecol Cancer 2008;18:312-7.

4. Hedman H, Lindstrom AK, Tot T, Stendahl U, Henriksson R, Hellberg D. LRIG2 in contrast to LRIG1 predicts poor survival in early-stage squamous cell carcinoma of the uterine cervix. Acta Oncol 2010;49:812-5.

5. Lindstrom AK, Asplund A, Hellberg D. Correlation between LRIG1 and LRIG2 expressions and expression of 11 tumor markers, with special reference to tumor suppressors, in CIN and normal cervical epithelium. Gynecol Oncol 2011;122:372-6.

6. Nilsson J, Starefeldt A, Henriksson R, Hedman H: LRIG1 protein in human cells and tissues. Cell Tissue Res 2003;312:65-71.

7. Zhou Y, Luoh SM, Zhang Y, Watanabe C, Wu TD, Ostland M, et al. Genome-wide identification of chromosomal regions of increased tumor expression by transcriptome analysis. Cancer Res 2003;63:5781-4.

8. Reifenberger G, Ichimura K, Reifenberger J, Elkahloun AG, Meltzer PS, Collins VP.Refined mapping of 12q13-q15 amplicons in human malignant gliomas suggests CDK4/SAS and MDM2 as independent amplification targets. Cancer Res 1996;56: 5141-5.

9. Benedet JL, Bender H, Jones H 3rd, Ngan HY, Pecorelli S. FIGO staging classifications and clinical practice guidelines in the management of gynecologic cancers. FIGO Committee on Gynecologic Oncology. Int J Gynaecol Obstet 2000;70:209-62.

10. Prasad CJ, Sheets E, Selig AM, McArthur MC, Crum CP. The binucleate squamous cell: histologic spectrum and relationship to low-grade squamous intraepithelial lesions. Mod Pathol 1993;6:313-7.

11. Uhlen M, Ponten F. Antibody-based proteomics for human tissue profiling. Mol Cell Proteomics 2005;4:384-93.

12. Lindstrom AK, Stendahl U, Tot T, Hellberg D. Associations between ten biological tumor markers in squamous cell cervical cancer and serum estradiol, serum progesterone and smoking. Anticancer Res 2007;27:1401-6.

13. Gustavsson I, Juko-Pecirep I, Backlund I, Wilander E, Gyllensten U. Comparison between the Hybrid Capture 2 and the hpVIR real-time PCR for detection of human papillomavirus in women with ASCUS or low grade dysplasia. J Clin Virol 2009;45:85-9.

14. Samir R, Asplund A, Tot T, Pekar G, Hellberg D. High-risk HPV infection and CIN grade correlates to the expression of c-myc, CD4+, FHIT, E-cadherin, Ki-67, and pl6INK4a. J Low Genit Tract Dis 2011;15: 280-6.

15. Hedman H, Nilsson J, Guo D, Henriksson R. Is LRIG1 a tumour suppressor gene at chromosome 3p14.3? Acta Oncol 2002;41:352-4.

16. Holmlund C, Haapasalo H, Yi W, Raheem 0, Brannstrom T, Bragge H, et al. Cytoplasmic LRIG2 expression is associated with poor oligodendroglioma patient survival. Neuropathology 2009;29:242-7.

17. Muller S, Lindquist D, Kanter L, FloresStaino C, Henriksson R, Hedman H, et al.
Expression of LRIG1 and LRIG3 correlates with human papillomavirus status and patient survival in cervical adenocarcinoma. Int J Oncol 2013;42:247-52.

18. Lindstrom AK, Tot T, Stendahl U, Syrjanen S, Syrjanen K, Hellberg D. Discrepancies in expression and prognostic value of tumor markers in adenocarcinoma and squamous cell carcinoma in cervical cancer. Anticancer Res 2009;29:2577-8.

19. Guo D, Nilsson J, Haapasalo H, Raheem O, Bergenheim T, Hedman $\mathrm{H}$, et al. Perinuclear leucine-rich repeats and immunoglobulin-like domain proteins (LRIG1-3) as prognostic indicators in astrocytic tumors. Acta Neuropathol 2006;111: 238-46.

20. Cai M, Han L, Chen R, Ye F, Wang B, Han F, et al. Inhibition of LRIG3 gene expression via RNA interference modulates the proliferation, cell cycle, cell apoptosis, adhesion and invasion of glioblastoma cell (GL15). Cancer Lett 2009;278:104-12.

21. Cai MJ, Xie RF, Han L, Chen RD, Wang BF, Ye F, et al. Effect of RNAi-mediated LRIG3 gene silencing on proliferation of glioma GL15 cells and expression of PCNA and Ki67. Ai Zheng 2009;28:1-4.

22. Cai M, Xie R, Han L, Chen R, Wang B, Ye F, et al. Effects of RNAi-mediated gene silencing of LRIG3 expression on cell cycle and survival of glioma cells. J Huazhong Univ Sci Technolog Med Sci 2009;29:88-93.

23. Yuan X, Bao S, Yang W, Ye Z. Effect of silencing LRIG3 gene on the proliferation and apoptosis of bladder cancer T24 cells. $\mathrm{J}$ Huazhong Univ Sci Technolog Med Sci 2011;31:220-5.

24. Brinton LA, Huggins GR, Lehman HF, Mallin K, Savitz DA, Trapido E, et al. Long-term use of oral contraceptives and risk of invasive cervical cancer. Int J Cancer 1986;38:39-44.

25. Zondervan KT, Carpenter LM, Painter R, Vessey MP. Oral contraceptives and cervical cancer - further findings from the 0xford Family Planning Association contraceptive study. Br J Cancer 1996;73:1291-7.

26. Samir R, Asplund A, Tot T, Pekar G, Hellberg D. Oral contraceptive and progestin-only use correlates to tissue tumor marker expression in women with cervical intraepithelial neoplasia. Contraception 2012;85: 88-93.

27. Hefler L, Grimm C, Tempfer C, Reinthaller A. Treatment with vaginal progesterone in women with low-grade cervical dysplasia: a phase II trial. Anticancer Res 2010;30: 1257-61.

28. Darwish A, Labeeb S, Galal M, Rashad H, Hassan S. Cervical changes associated with progestagen-only contraceptives: a team approach. Contraception 2004;69:121-7. 